\title{
NUMERICAL RANGE ESTIMATES FOR THE NORMS OF ITERATED OPERATORS
}

\author{
by M. J. CRABB
}

(Received 8 February, 1969)

Let $X$ be a complex normed space, with dual space $X^{\prime}$, and $T$ a bounded linear operator on $X$. The numerical range $V(T)$ of $T$ is defined as $\left\{f(T x): x \in X, f \in X^{\prime},\|x\|=\|f\|=f(x)=1\right\}$. Let $|V(T)|$ denote sup $\{|\lambda|: \lambda \in V(T)\}$. Our purpose is to prove the following theorem.

THEOREM. $\quad\left\|T^{n}\right\| \leqq n !\left(\frac{e}{n}\right)^{n}|V(T)|^{n} \quad(n=1,2, \ldots)$.

From the proof of Stirling's formula, it is known that

$$
\frac{n ! e^{n}}{n^{n}} \leqq e n^{\frac{1}{2}} \quad(n=1,2, \ldots)
$$

The estimate for $\left\|T^{n}\right\|$ given in the present theorem is therefore very much better than the estimate $\left\|T^{n}\right\| \leqq e^{n}|V(T)|^{n}$ given by the case $n=1$.

When $X$ is a complex Hilbert space, $V(T)=\{(T x, x):\|x\|=1\}$. In this case, Berger [1] proves that $|V(T)| \leqq 1$ implies that $\left|V\left(T^{n}\right)\right| \leqq 1$, and so $\left\|T^{n}\right\| \leqq 2$, for positive integers $n$. An elementary proof of this is given by Pearcy [8]. For a general normed space, $V(T)$ is the union of all possible numerical ranges $W(T)$ in the sense of Lumer [6]. For each such $W(T)$, $|W(T)|=|V(T)|$, and so we may replace $V$ by $W$ in (1). The theorem for the case $n=1$ was proved by Bohnenblust and Karlin [3]; a simplified proof was given by Glickfeld [5], and the present result is based on his argument.

We shall require the following elementary result from Lumer [6].

LEMma. Let $T$ be a bounded linear operator on a Banach space, with $|V(T)|<1$. Then $(I-T)^{-1}$ exists, $\left\|(I-T)^{-1}\right\| \leqq(1-|V(T)|)^{-1}$, and $\rho(T) \leqq|V(T)|$, where $\rho(T)$ denotes the spectral radius of $T$.

Proof of Theorem. By [6], we have

$$
\sup \operatorname{Re} V(T)=\lim _{\alpha \rightarrow 0^{+}} \frac{\|I+\alpha T\|-1}{\alpha}
$$

and therefore $|V(T)|$ is unchanged if $X$ is replaced by its completion. We assume therefore that $X$ is complete.

Let $\omega_{k}(k=1,2, \ldots, m)$ be the $m$ th roots of unity. Let $n$ and $p$ be positive integers. Assume first that $|V(T)|=\mu<1$, and that $m>n$. Then 


$$
\begin{aligned}
\frac{1}{m} \sum_{k=1}^{m} \omega_{k}^{n}\left(I-\omega_{k}^{-1} T\right)^{-p} & =\frac{1}{m} \sum_{k=1}^{m} \omega_{k}^{n}\left\{I+p \omega_{k}^{-1} T+\ldots+\frac{p(p+1) \ldots(p+n-1)}{1.2 \ldots n} \omega_{k}^{-n} T^{n}+\ldots\right\} \\
& =\frac{p(p+1) \ldots(p+n-1)}{1.2 \ldots n} T^{n}+\frac{p(p+1) \ldots(p+n+m-1)}{1.2 \ldots(n+m)} T^{n+m}+\ldots
\end{aligned}
$$

By the lemma,

$$
\left\|\left(I-\omega_{k}^{-1} T\right)^{-1}\right\| \leqq(1-\mu)^{-1} .
$$

Therefore

$$
\left\|\left(I-\omega_{k}^{-1} T\right)^{-p}\right\| \leqq(1-\mu)^{-p},
$$

and

$$
\left\|\frac{1}{m} \sum_{k=1}^{m} \omega_{k}^{n}\left(I-\omega_{k}^{-1} T\right)^{-p}\right\| \leqq(1-\mu)^{-p} .
$$

Letting $m \rightarrow \infty$, we deduce that

$$
\frac{p(p+1) \ldots(p+n-1)}{1.2 \ldots n}\left\|T^{n}\right\| \leqq(1-\mu)^{-p}
$$

If $\mu=0$, (2) gives $p\|T\| \leqq 1(p=1,2, \ldots)$, so that $T=0$. So assume that $\mu \neq 0$.

Now let $T$ be any bounded linear operator on $X$, and apply (2) to $n T /(p+1) \mu$ for $p>n-1$. Then

$$
\left\|T^{n}\right\| \leqq \frac{1.2 \ldots n}{p(p+1) \ldots(p+n-1)}\left(\frac{p+1}{n}\right)^{n}\left(\frac{p-n+1}{p+1}\right)^{-p} \mu^{n} .
$$

Letting $p \rightarrow \infty$, we have

$$
\left\|T^{n}\right\| \leqq n !\left(\frac{e}{n}\right)^{n} \mu^{n}
$$

Remark. We do not know of any operator $T$ such that $|V(T)| \leqq 1$ and $\left\{\left\|T^{n}\right\|\right\}$ is unbounded. It is quite easy to prove that, if $T$ is an operator on a finite-dimensional space, or, more generally, is a meromorphic operator (Taylor [9], Caradus [4]), then $\left\{\left\|T^{n}\right\|\right\}$ is bounded whenever $|V(T)| \leqq 1$.

To prove this, let $T$ be a bounded linear meromorphic operator with $|V(T)|=1$. Let $\operatorname{sp}(T)$ denote the spectrum of $T$. Suppose that $\lambda \in \operatorname{sp}(T)$ with $|\lambda|=1$. Then there exists an idempotent $P$ such that $T P=P T,(\lambda I-T) P$ is nilpotent, and $(\lambda I-T)(I-P)$ is invertible in $(I-P) X$. Since $\lambda$ is a boundary point of the convex hull of $V(T)$, Theorem 4 of Nirschl and Schneider [7], extended to the case of linear operators on general normed linear spaces, is applicable. This shows that $(\lambda I-T)^{2} x=0$ implies that $(\lambda I-T) x=0$. It follows that $(\lambda I-T) P=0$. Therefore

$$
T=T P+T(I-P)=\lambda P+T(I-P) .
$$

Since the non-zero points of $\operatorname{sp}(T)$ are isolated, $T$ may therefore be written

$$
T=\sum_{i=1}^{m} \lambda_{i} P_{i}+S
$$


where $\left|\lambda_{i}\right|=1, P_{i}^{2}=P_{i}, P_{i} S=S P_{i}=0, P_{i} P_{j}=0(i \neq j)$, and $\rho(S)<1$. Then

$$
T^{n}=\sum_{i=1}^{m} \lambda_{i}^{n} P_{i}+S^{n}
$$

so that $\left\{\left\|T^{n}\right\|: n=1,2, \ldots\right\}$ is bounded.

Added in proof. The author has found an example of a non-zero operator $T$ for which equality holds in (1) for every integer $n$; details of this will be published elsewhere.

\section{REFERENCES}

1. C. Berger, On the numerical range of an operator, Bull. Amer. Math. Soc. (to appear).

2. C. Berger and J. Stampfli, Mapping theorems for the numerical range, Amer. J. Math. 89 (1967), 1047-1055.

3. H. Bohnenblust and S. Karlin, Geometrical properties of the unit sphere in Banach algebras, Ann. of Math. 62 (1955), 217-229.

4. S. R. Caradus, On meromorphic operators, I, Canad. J. Math. 19 (1967), 723-736.

5. B. Glickfeld, On an inequality in Banach algebra geometry and semi-inner-product spaces, Notices Amer. Math. Soc. 15 (1968), 339-340.

6. G. Lumer, Semi-inner-product spaces, Trans. Amer. Math. Soc. 100 (1961), 29-43.

7. N. Nirschl and H. Schneider, The Bauer field of values of a matrix, Numer. Math. 6 (1964), 355-365.

8. C. Pearcy, An elementary proof of the power inequality for the numerical radius, Michigan Math. J. 13 (1966), 289-291.

9. A. E. Taylor, Mittag-Leffler expansions and spectral theory, Pacific J. Math. 10, 3 (1960), 1049-1066.

\section{UNIVERSITY OF EDINBURGH}

